\title{
Genomic instability links diet to cancer
}

DOI:

10.1038/nrg2213
A genetic screen in yeast has found a surprising link between vitamin B6 deficiency and increased genome instability, a hallmark of cancerous cells. This study gives new insights into the causes of genetic aberrations, and provides a possible mechanistic explanation for epidemiological evidence that suggests a link between micronutrient deficiencies and cancer risk.

When faithful DNA replication is disrupted, genetic lesions are created. If the DNA-repair pathways that correct these lesions are faulty, illegitimate repair can result in gross chromosomal rearrangements (GCRs) such as translocations, amplifications, inversions and deletions. In particular, breakinduced replication (BIR), a type of homologous recombination repair, is thought to be a major mechanism by which GCRs occur.

Kanellis and colleagues engineered a Saccharomyces cerevisiae strain that includes a GCR reporter located at a chromosomal position at which rearrangements take place by BIR. In a genome-wide screen,

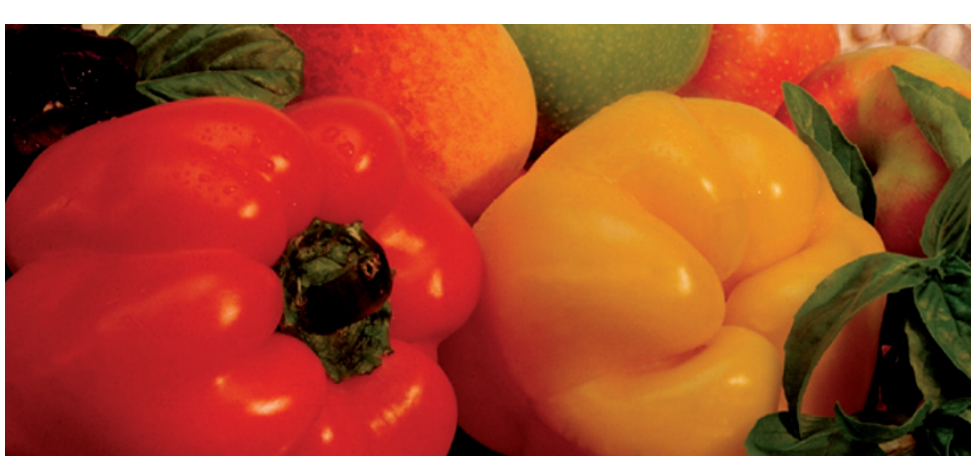

the authors picked out BUD16 as a potent suppressor of GCR, and alignment studies revealed that this gene encodes a putative pyridoxal kinase (Pdxk), an enzyme that is crucial for the metabolism of vitamin B6 to produce pyridoxal $5^{\prime}$ phosphate (PLP), the biologically active form. Strikingly, bud16 16 mutant cells have a 124 -fold increased GCR rate compared with wild-type cells, coinciding with a reduction in PLP levels to $1.8 \%$.

Rad52 is essential for homologous recombination and hence DNA repair. bud16 $\mathrm{rad} 52 \Delta$ double mutants showed synthetic sickness and poor viability, suggesting that bud16 $\Delta$ cells have high levels of DNA disruption during replication, and rely on Rad52-mediated repair for survival. Furthermore, fluorescence microscopy showed that, after budding, Rad52 foci (or 'repair centres') were present in $57-75 \%$ of bud $16 \Delta$ cells, compared with $2-21 \%$ in wild-type cells.

But do these symptoms of replication stress necessarily result from
PLP depletion? By interfering with

- depletion of metabolites resulting from overproliferation of cancerous cells might be sensed by the cells as replication stress, activating damage-response pathways to bring about senescence.

Carrie Patis

ORIGINAL RESEARCH PAPER Kanellis, P. et al. A screen for suppressors of gross chromosomal rearrangements identifies a conserved role for PLP in preventing DNA lesions. PLoS Genet. 3, e134 (2007) 